\title{
C3 Glomerulonephritis Associated with Monoclonal Gammopathy of Renal Significance
}

\author{
Glomerulonefrite C3 Associada a Gamopatia \\ Monoclonal de Significado Renal
}

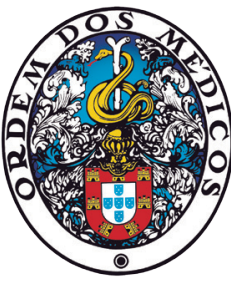

\author{
Inês GOMES-ALVES $\square^{1}$, Inês CASTRO-FERREIRA ${ }^{2}$ \\ Acta Med Port 2021 May;34(5):372-377 - https://doi.org/10.20344/amp.13823
}

\begin{abstract}
Introduction: Monoclonal gammopathy of renal significance (MGRS) is described as a hematologic condition characterized by nephrotoxic monoclonal proteins produced by a non-malignant B-cell or plasma cell clone. Nevertheless, MGRS can cause serious renal lesions, leading to high morbidity. In C3 glomerulonephritis, a monoclonal protein can cause renal damage indirectly. Acting as an autoantibody, the protein cannot be detected in the kidney biopsy, promoting the dysregulation of the alternative pathway of the complement system.
\end{abstract}

Material and Methods: This non-systematic review was based on a comprehensive search in databases and scientific journals, such as PubMed, Nature Reviews Nephrology and Kidney International, including the terms 'C3 Glomerulonephritis' and 'Monoclonal gammopathy of renal significance'. We review the pathophysiology, presentation, diagnosis, differential diagnosis and treatment of C3 glomerulonephritis associated with MGRS.

Discussion: With the increasing understanding of the complex interaction between monoclonal gammopathy and renal damage, such as C3 glomerulonephritis, it becomes clear that an early recognition is crucial, as Ig-directed therapy might improve outcomes. In this context, and in order to maximize the chance of a correct diagnosis, renal biopsy is mandatory to determine the exact nature of the lesion, and the severity of renal disease.

Conclusion: It is important to make an early diagnosis of MGRS-associated C3 glomerulonephritis in order to prevent not only the progression to a hematological malignancy, but also end-stage renal disease.

Keywords: Glomerulonephritis; Monoclonal Gammopathy of Renal Significance

\section{RESUMO}

Introdução: A gamopatia monoclonal de significado renal (MGRS) é descrita como uma doença hematológica caracterizada pela existência de proteínas monoclonais nefrotóxicas produzidas por um clone não maligno de células B ou plasmócitos. A MGRS pode causar lesões renais graves, levando a elevada morbilidade. Na glomerulonefrite C3, a proteína monoclonal pode causar indiretamente lesão renal. A proteína atua como auto-anticorpo, não sendo detetada na biópsia renal, promovendo a desregulação da via alternativa do complemento.

Material e Métodos: Esta revisão não sistemática foi baseada numa pesquisa abrangente com recurso a base de dados e revistas científicas, como a PubMed, Nature Reviews Nephrology e Kidney International, utilizando os termos 'Glomerulonefrite C3' e 'Gamopatia monoclonal de significado renal'. Apresentamos uma revisão da fisiopatologia, apresentação clínica, diagnóstico, diagnóstico diferencial e tratamento de glomerulonefrite C3 associado a MGRS

Discussão: Com a crescente compreensão da complexa interação entre a gamopatia monoclonal e a lesão renal, como é exemplo a glomerulonefrite $\mathrm{C} 3$, torna-se claro que um reconhecimento precoce é crucial, dado que a terapia dirigida à lg pode melhorar o resultado. Neste contexto, para maximizar a probabilidade de um diagnóstico correto, uma biópsia renal é necessária para determinar a natureza exata da lesão e a severidade da doença renal.

Conclusão: É importante realizar um diagnóstico precoce de glomerulonefrite G3 associada a MGRS de modo a prevenir não apenas a progressão para uma neoplasia hematológica, mas também para doença renal terminal.

Palavras-chave: Gamopatia Monoclonal de Significado Renal; Glomerulonefrite

\section{INTRODUCTION}

Monoclonal gammopathy of undetermined significance (MGUS) is the most common plasma cell disorder. The prevalence in the general population is about $0.7 \%$ and increases with age, to $3.2 \%, 5.3 \%$ and $7.5 \%$ in people older than 50 years, 70 years and 85 years, respectively. It is also estimated to be more prevalent in men (4.0\%) compared to women $(2.7 \%){ }^{1}$

MGUS is defined by monoclonal immunoglobulin (lg) below $3 \mathrm{~g} / \mathrm{dL}$, plasma cells in the bone marrow below $10 \%$, and the absence of signs or symptoms related with multiple myeloma (MM) (CRAB: hypercalcemia, renal insufficiency, anemia and bone lesions) or other lymphoproliferative malignancies. ${ }^{2}$ It is a pre-malignant precursor, with a progression rate to $\mathrm{MM}$ or related malignant neoplasm of $1 \%$ per year, and this annual risk of progression is not affected by age or duration of the pre-malignant precursor. ${ }^{3}$

As a pre-malignant disorder, treatment of MGUS is not recommended until progression to MM.

However, the recognition of potential renal involvement led to the revision of the therapeutic approach, not previously considered. ${ }^{4}$

The term 'monoclonal gammopathy of renal significance'

1. Faculty of Medicine. University of Porto. Porto. Portugal.

2. Department of Nephrology. Faculty of Medicine. University of Porto. Porto. Portugal.

$\triangle$ Autor correspondente: Inês Gomes-Alves. iiines.alves@gmail.com

Recebido: 31 de março de 2020 - Aceite: 16 de junho de 2020 - First published: 05 de abril 2021 - Online issue published: 03 de maio de 2021 Copyright $\odot$ Ordem dos Médicos 2021 
(MGRS) was introduced in 2012 and updated in 2017 by the International Kidney and Monoclonal Gammopathy Research Group (IKMG). Nowadays, MGRS is described as a hematologic condition characterized by nephrotoxic monoclonal proteins, produced by a non-malignant B-cell or plasma cell clone, that does not meet any hematologic criteria for therapy targeting the underlying clonal disorder. ${ }^{5}$ The prevalence of MGRS is around $0.32 \%$ and $0.53 \%$ in people older than 50 and 70 years old, respectively. ${ }^{6}$

MGRS can be associated with a variety of renal disorders. However, no renal lesion is specific of any hematologic disorder. The nephrotoxic monoclonal immunoglobulin (Ig) or fragment can affect any structure in the renal parenchyma, and the pattern of renal lesion is mostly determined by the intrinsic structural and physicochemical characteristics of the monoclonal protein (intact monoclonal immunoglobulins or immunoglobulin light chains), rather than by the rate of production, and clone features. ${ }^{5,7}$ On the other hand, the kidneys receive approximately $20 \%$ of the cardiac output, and have a unique environment where its chemical properties might change the monoclonal protein making it more toxic, and therefore, making kidneys more susceptible to damage. 8,9 There are essentially two types of mechanisms of renal injury based on the detection of monoclonal protein: direct and indirect. The most important mechanism is the direct one, characterized by the presence of monoclonal Ig deposits, which can be formed by the full or parts of the monoclonal immunoglobulin (heavy or light chains) or by various products of aggregation. ${ }^{5}$ In the indirect mechanism, there are no Ig deposits, and monoclonal Ig acts as an autoantibody, dysregulating the alternative pathway of the complement system.

The best example of a MGRS-associated disorder with absent or scant monoclonal Ig deposition is C3 Glomerulopathy, more precisely C3GN and Dense Deposits Disease (DDD). ${ }^{6,10}$

In fact, a high proportion (reaching $65.1 \%$ ) of patients older than 50 years of age with the diagnosis of C3 glomerulopathy have detectable levels of serum monoclonal $\mathrm{lg}$, which means a prevalence of monoclonal $\mathrm{lg}$ in this group 16 times higher compared with the general population $(4.2 \%))^{1,11}$

C3 glomerulopathy is a histopathological diagnosis which encompasses a group of rare renal diseases affecting about 1 to 2 per million people worldwide and is characterized by dysregulation of the alternative pathway of the complement system, leading to complement C3 deposition in the glomerulus, with sparse immunoglobulin deposition. Additionally, terminal pathway dysregulation might also occur, especially in C3GN. ${ }^{10,12}$

This abnormal control of the alternative pathway may be driven by acquired or genetic changes. Genetic causes are less frequent and include mutations that result either in loss of function in genes responsible for regulatory proteins or in gain of function in activator proteins. Acquired factors include the presence of autoantibodies towards regulatory proteins. The most common are the C3 nephritic factors, which stabilize C3 convertase, increasing its half-life, but other autoantibodies have been identified such as C5 nephritic factors (which target $\mathrm{C} 3 \mathrm{bBbC} 3 \mathrm{~b}$ ), C4 nephritic factors (towards $\mathrm{C} 4 \mathrm{~b} 2 \mathrm{a}$ ), factor $\mathrm{H}$ and factor $\mathrm{B}$ autoantibodies. ${ }^{10,13}$

In this review we are going to focus on C3GN associated with MGRS.

\section{MATERIAL AND METHODS}

We performed a comprehensive search in databases and scientific journals, such as PubMed, Nature Reviews Nephrology and Kidney International, including the terms 'C3 Glomerulonephritis' and 'Monoclonal gammopathy of renal significance' until December 2019. A total of 58 articles were initially selected, with subsequent exclusion of 28 (language not in English, and/or inclusion of glomerulonephritis other than C3GN). We present a review of the pathophysiology, presentation, diagnosis, differential diagnosis, and treatment of C3GN associated with MGRS.

\section{Diagnostic approach \\ Presentation}

The typical presentation of C3GN is a nephritic syndrome, characterized by edema, hematuria, proteinuria, azotemia and hypertension, with high risk for the development of end-stage renal disease (ESRD).

C3GN is a rare disease that affects children and adults, and C3GN associated with MGRS is even more exceptional.

MGUS increases with age, being around $3.2 \%$ in people older than 50 years old and reaching approximately $31.2 \%$ for patients with C3GN with the same age group. .,14 $^{1,1}$

Therefore, patients aged more than 50 years with features of C3GN on renal biopsy, should be evaluated for the presence of a monoclonal gammopathy.

\section{Renal biopsy}

A renal biopsy is mandatory to assess the diagnosis. It is indicated for clarification of the etiology of a nephritic syndrome, as well as in cases of MGUS with suspected renal involvement, for instance because of the presence of isolated proteinuria or nephritic syndrome.

Moreover, renal biopsy provides valuable additional information such as the severity of the renal damage, through the activity and chronicity of the lesions observed. ${ }^{15}$ This procedure remains essential even in patients with advanced renal disease in whom renal transplantation is intended, since the disease recurs in the allograft in the absence of control of the underlying clone. ${ }^{16}$

On light microscopy, C3GN usually shows a membranoproliferative pattern but can also show mesangial proliferative glomerulonephritis. The electron microscopy (EM) offers valuable supplementary information, regarding the structure of the deposits and their location. In C3GN, it typically reveals ill-defined electron dense deposits in the subendothelial space, subepithelial space and/or mesangium. This procedure is critical for the distinction of the 
other forms of C3 glomerulopathy associated with MGRS, the DDD, which shows highly electron dense 'sausage like' deposits that segmentally infiltrate the glomerular basement membrane lamina densa. ${ }^{7}$

Interestingly, humps are common in both, C3GN and DDD on light microscopy. ${ }^{7}$

The immunofluorescence microscopy is positive for granular C3 deposits, of at least two orders of magnitude more intense than any other immune reactants but negative for Ig (either heavy or light chains), and components of the classical or lectin pathways (particularly of $\mathrm{C} 1 \mathrm{q}$ and $\mathrm{C} 4$ ). This pattern is known as glomerulonephritis with dominant C3. ${ }^{10,12,15}$

Laser microdissection and mass spectrometry are useful to confirm the presence of C3 deposition, especially C3dg, which is a breakdown product, as well as small amounts of C5, C6, C7, C8, C9 and the complement factor-H-related proteins (FHR1-FHR5). Moreover, it confirms the absence of $\lg .10,12,15$

\section{Complement evaluation}

Investigation of the complement system should be done in all patients with a renal biopsy that is suggestive of C3GN.

As a result of the uncontrolled activation of the alternative pathway and $\mathrm{C} 3$ glomerular deposition, the serum level of C3 can be decreased and C3 breakdown products elevated (Fig. 1). The serum level of $\mathrm{C} 4$ is frequently normal but might be low in some patients. In addition, factor $B$ and factor $\mathrm{H}$ might also be low. ${ }^{15}$

As a result of the terminal pathway dysregulation that could also occur in C3GN, serum C5 and C5b-9 levels might be low and elevated, respectively. However, these measures imply a sophisticated and difficult technique that is not widely available. ${ }^{15}$

Since complement dysregulation can be driven by both acquired and genetic factors, a detailed assessment of these forms is crucial.

As acquired drivers, C3 and C5 nephritic factors are the most frequent in $\mathrm{C} 3 \mathrm{GN}$. In addition, both factor $\mathrm{H}$ and factor B autoantibodies, as well as $\mathrm{C} 4$ nephritic factors should also be tested by functional assays of complement activity, especially if C3GN associated with MGRS is suspected. ${ }^{11}$ C3 nephritic factors exist approximately in 50\% of patients with C3GN, but can also appear in healthy individuals, as well as in other glomerulopathies. ${ }^{11}$ Therefore, even though it can act as a driver, it might not be sufficient to cause the disease, highlighting the relevance of screening other possible drivers. ${ }^{17}$

Genetic screening is indicated for the assessment of the etiology of C3GN. Nevertheless, concomitant genetic factors are infrequent in C3GN associated with MGRS, reaching approximately only $7 \%$ of patients. ${ }^{18}$

It should particularly include mutations in the complement regulatory protein factor $\mathrm{H}, \mathrm{I}$, and CD46. Furthermore, in patients with negative $\mathrm{C} 3$ nephritic factor, complement factor $\mathrm{H}$ mutations should not be missed as well. ${ }^{12}$

Conversely, gain of function mutations in activators proteins (factor B and C3), are infrequent. ${ }^{12}$

\section{Screening for monoclonal immunoglobulin}

In C3GN associated with MGRS, the monoclonal Ig can be a trigger, and might act as an autoantibody against alternative pathway regulating proteins, acting as C3 nephritic factor or a factor-H antibody. ${ }^{14}$ With this in mind, screening for monoclonal lg must be done, especially in patients over 50 years old. The screening includes serum and urine

Alternative pathway

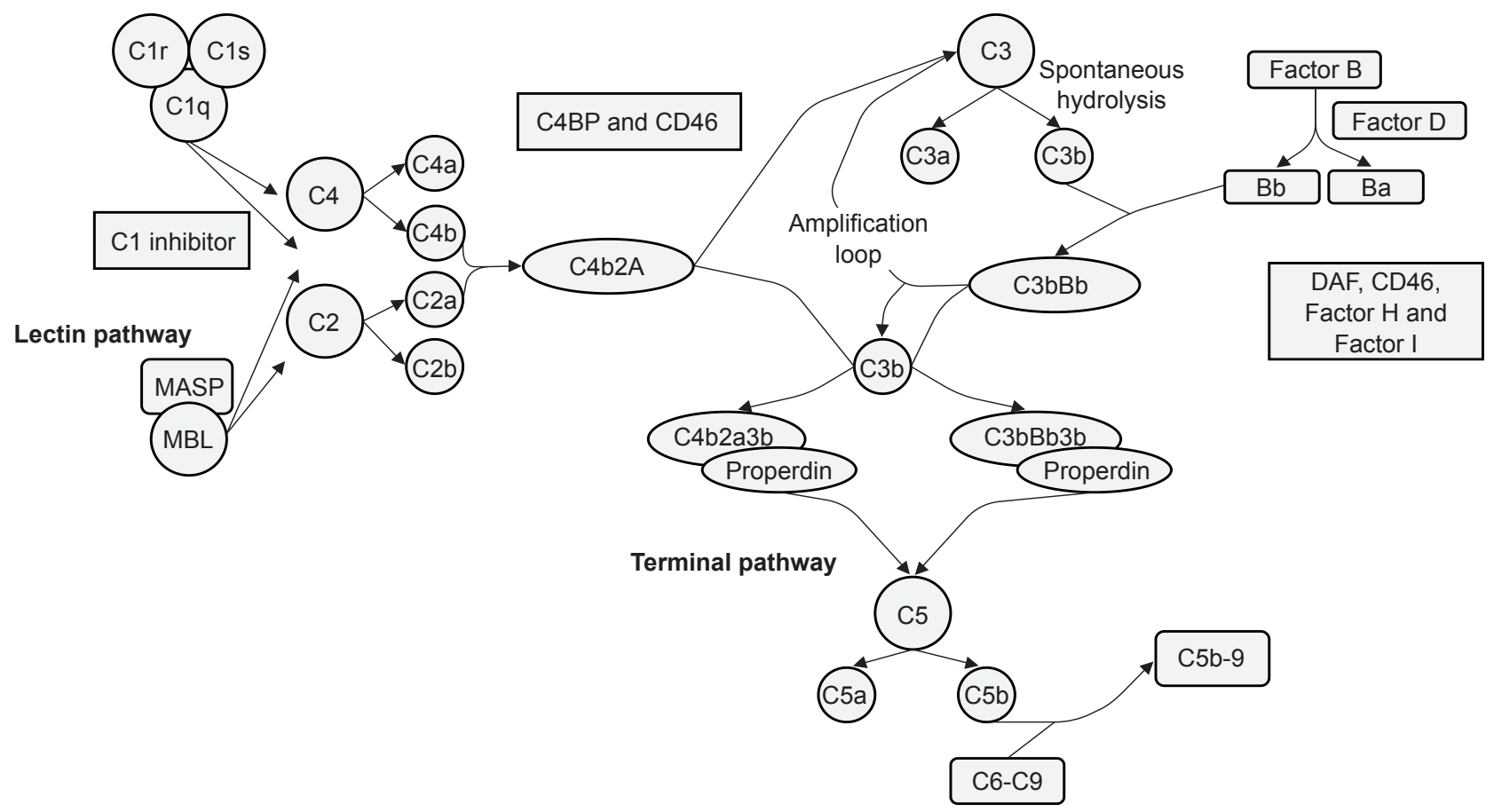

Figure 1 - Complement cascade 
protein electrophoresis, immunofixation electrophoresis, as well as serum-free light chain (sFLC) immunoassay. ${ }^{3,14,19}$ Serum protein electrophoresis is an inexpensive and rapid technique that allows the quantification of the M-component, which is important for the diagnosis, and for the evaluation of treatment response. However, it may not detect low levels of monoclonal $\mathrm{lg}$, a presentation not uncommon in MGRS. ${ }^{20}$ On the other hand, only free light chains are filtered by healthy glomerulus, and consequently urine protein electrophoresis is the least sensitive test. Despite that, it may help to identify the pattern of renal injury through the detection of the level of total protein, albumin and globular proteins, details that can be important for the diagnosis and treatment monitoring. ${ }^{5,21}$ Serum and urine immunofixation are more sensitive to detect small clones, contributing to a more precise evaluation of the treatment response. The serum FLC assay has high sensitivity and uses polyclonal antibodies specific to epitopes (which are only uncovered when light chains are free) in order to measure kappa, lambda lights chains, and its ratio. If the value of the ratio falls outside of the normal range, that can be due to the overproduction of one of the FLC, and therefore a monoclonal FLC is identified. Moreover, if the glomerular filtration rate is decreased, the serum concentration of FLC will increase, widening the ratio estimates. In order to increase the specificity, without losing sensitivity, adapted reference range values should be used.

Time-of-flight mass spectrometry and urinary exosomes are two new techniques with the ability to detect a very low amount of monoclonal $\mathrm{lg}$ and distinguishing nephrotoxic from non-nephrotoxic monoclonal lights chains, respectively. ${ }^{20}$

\section{Clonal identification}

After the documentation of the monoclonal protein, the next step should be the identification and characterization of the clonal population of cells. In all patients a detailed hematologic evaluation should be carried out. This evaluation requires a sample of bone marrow, peripheral blood, as well as lymph nodes in some cases. The bone marrow biopsy or aspiration are usually enough to detect the clone. The type of underlying clone can be easily identified by morphological assessment. Nevertheless, additional studies may be necessary to complement the evaluation. On bone marrow biopsy, immunohistochemical staining for CD138 or CD5, CD10 and CD20 allows the identification of plasma cells and $\mathrm{B}$ cells population, respectively. Fluorescence in situ hybridization (FISH) can demonstrate restricted production of the monoclonal Ig, and flow cytometry can also be helpful in bone marrow aspirates, lymph nodes or peripheral blood. If no plasma cell clone is found on bone marrow or the paraprotein is $\operatorname{lgM}$, further imaging studies to find the B-cell clone should be performed, which include conventional radiography, computer tomography scan (CT) of the chest, abdomen and pelvis and/ or 18-fludeoxyglucose positron emission tomography (PET)/CT. Any suspicious lymph nodes found should undergo a biopsy. ${ }^{5,7,22,23}$ Furthermore, clone identification and characterization are critical not only in terms of diagnosis, but also in terms of therapeutic approach.

\section{Diagnostic challenges}

Besides the differential diagnosis between DDD and C3GN, provided by EM findings, other conditions should be excluded.

Post-infectious glomerulonephritis (PIGN) is a common misleading diagnosis. ${ }^{24}$

About $30 \%$ of PIGN can have isolated C3 deposits with no Ig, particularly during the late stage. But distinction from C3GN can be made as the clinical and laboratory findings of PIGN are self-limited, with normalization usually within 8 weeks of resolution of the infection. ${ }^{10}$ Therefore, progressive renal impairment, persistent hypocomplementemia, hematuria and proteinuria after 12 weeks should raise the suspicion of C3GN, and a repeat renal biopsy is required. Of note, some studies suggest that some cases of PIGN might take longer to resolve, and are classified as 'atypical'. Furthermore, when an infection occurs, it can act as a trigger to the alternative pathway, which normalizes with the resolution of the infection. However, in patients with a defect of the alternative pathway, the infection may unmask or exacerbate this abnormality, and consequently the clinical and laboratory features may have a slower rate of resolution. In these cases, an overlap between C3GN and 'atypical' PIGN in biopsy findings might be found, underlining the importance of a detailed evaluation of the clinical history to achieve the correct diagnosis. ${ }^{24}$

Approximately $5 \%-10 \%$ of patients with monoclonal gammopathy in whom renal biopsy shows a C3GN will, in fact, have a membranoproliferative glomerulonephritis with masked monoclonal deposits. ${ }^{23}$ In order to avoid misdiagnosis, all patients diagnosed with C3GN associated with monoclonal gammopathy should undergo a pronase immunofluorescence. ${ }^{25}$ This technique uses a paraffin-embedded tissue with an antigen-retrieval step by pronase digestion, which helps to unmask monoclonal deposits. In spite of being more sensitive as an unmasking technique, it has poor sensitivity for grading C3 staining intensity when compared with immunofluorescence on frozen tissue. ${ }^{25,26}$ Along with no masked Ig deposits on pronase immunofluorescence, the absence of $\mathrm{C} 4 \mathrm{~d}$ staining can also be helpful to exclude immune complex glomerulonephritis. ${ }^{10,27}$

In conclusion, the diagnosis of C3GN associated with MGRS is a diagnosis of exclusion that requires a renal biopsy, albeit with some pitfalls for misdiagnosis. Achieving the right diagnosis requires a high index of suspicion during all the steps of the evaluation.

\section{Treatment}

The best treatment for C3GN associated with MGRS has not been established. However, after the diagnosis of $\mathrm{C} 3 \mathrm{GN}$, all patients require renoprotective treatment, adequate nutrition, and modification of unhealthy lifestyle behaviors. Renoprotective treatment includes angiotensin-converting enzyme inhibitors or angiotensin-receptor 
blockers as first-line therapy for proteinuria and hypertension, and lipid-lowering agents in case of dyslipidemia. Nevertheless, this treatment may not be sufficient to prevent ESRD. However, in addition to immunosuppressant treatment, it contributes to better renal outcomes.

Despite the absence of specific guidelines for the treatment of C3GN associated with MGRS, there is some evidence that Ig-target therapy is useful in delaying the progression of renal disease, highlighting the correlation between the reduction of monoclonal $\mathrm{lg}$ and better renal outcomes. $^{7,11}$ Moreover, successful treatment favors the risk reduction of recurrence of $\mathrm{C} 3 \mathrm{GN}$ after renal transplantation. Treatment should be initiated even in patients with advanced renal disease, as long as kidney transplantation is being considered. ${ }^{21}$ The type and nature of monoclonal protein is important for the selection of Ig-targeted agent, but the clinician also has to take into account histological features of C3GN (balance between active and chronic lesions), renal function, and potential toxicity, which can preclude some of them. ${ }^{28}$

Bortezomib is a proteasome inhibitor preferred in MGRS with no need of dose adjustment in patients with chronic kidney disease (CKD), including in patients on dialysis. By itself, bortezomib can achieve a response rate between $30 \%$ and $50 \%$, and when associated with dexamethasone and cyclophosphamide, the rate can increase up to $94 \%{ }^{8,22}$ A clone with high expression of CD20, particularly a B cell clone, rituximab, an anti-CD20 monoclonal antibody, is a drug not to be overlooked, and full doses can also be given in CKD without the need for dose adjustments. ${ }^{22}$ Among cytotoxic therapies, cyclophosphamide and melphalan, whose target are both plasma and B cells, are also optimal choices in MGRS. The first one is used more often, and is typically given at full doses, without hematologic stem cell toxicity. Melphalan is usually considered in candidates for autologous stem cell transplantation, but it requires dose adjustment in patients with CKD stage $\geq 3$. Another option is bendamustine. Thalidomide is an immunomodulatory drug with activity against plasma and B cells, albeit with potential neurotoxicity. ${ }^{29}$ Despite activity against $B$ cells, purine analogs should be avoided due to the need of renal dose adjustment, and high risk for the development of adverse effects. ${ }^{22,28}$ Furthermore, in patients with a plasma cell disorder, autologous stem cell transplant can be beneficial as a complementary therapy, helping to achieve a deeper and sustained or even a complete hematological response, which is essential to reduce the risk of recurrence, particularly after renal transplant. ${ }^{21}$

As a consequence of the lack of standardized therapies, treatment options should be carefully evaluated in each case in terms of risk/ benefit ratio. ${ }^{28}$ Nevertheless, patients should be followed-up regularly by a nephrologist and a hematologist not only to prevent progression of renal damage, but also to detect early recurrence of monoclonal gammop- athy and potential progression to a hematologic malignant disease, like MM. ${ }^{30}$

\section{DISCUSSION}

The best example of a case of MGRS-associated disorder without deposits of monoclonal $\mathrm{lg}$ is C3GN, where monoclonal protein causes renal damage indirectly through continuous activation of the alternative pathway of the complement system. This glomerulonephritis typically presents with hematuria, proteinuria, and renal impairment. Besides a careful anamnesis, physical examination and biochemical tests, a renal biopsy is required not only to determine the nature and severity of the lesion, but also to confirm the diagnosis. Immunofluorescence microscopy must show a predominant $\mathrm{C} 3$ staining, and EM findings are essential to differentiate C3GN from DDD. Complement evaluation should also be done in all patients, and should include measurement of complement proteins, genetic testing, screening for autoantibodies and functional assays of complement activity. In this evaluation, C3 serum levels are typically low because of the dysregulation of the alternative pathway, C5 and C5b-9 serum levels might be low and elevated respectively, as a result of the uncontrolled activation of the terminal pathway that can also occur.

Given the evidence that the incidence of monoclonal gammopathy in the general population increases with age, particularly in C3GN where it reaches a significant proportion in patients older than 50 years, screening for monoclonal $\mathrm{lg}$ is highly recommended, and clonal identification should follow monoclonal protein documentation. Pronase immunofluorescence should also be done to exclude unmasked Ig deposits, in order to avoid misdiagnosis with other membranoproliferative glomerulonephritis, specifically those that are immune complex mediated.

There are no standardized guidelines for treatment, and effective therapy requires an individualized approach, evaluating the risk/benefit ratio. Besides general renoprotective measures, treatment should focus on the hematologic disease given the correlation between hematologic response and renal outcomes, in addition to the prevention of recurrence after renal transplantation.

\section{CONCLUSION}

C3GN associated with MGRS is a diagnosis of exclusion, requiring a high index of suspicion during all the steps of evaluation. Clinical awareness is required in order to provide an early diagnosis, avoid delays in treatment initiation, and improve outcomes.

\section{CONFLICTS OF INTEREST}

The authors declare they have no conflict of interest.

\section{FUNDING SOURCES}

No funding was received for this study. 


\section{REFERENCES}

1. Kyle RA, Therneau TM, Rajkumar SV, Larson DR, Plevak MF, Offord JR, et al. Prevalence of monoclonal gammopathy of undetermined significance. N Engl J Med. 2006;354:1362-9.

2. van de Donk NW, Palumbo A, Johnsen HE, Engelhardt M, Gay $\mathrm{F}$, Gregersen $\mathrm{H}$, et al. The clinical relevance and management of monoclonal gammopathy of undetermined significance and related disorders: recommendations from the European Myeloma Network. Haematologica. 2014;99:984-96.

3. Kyle RA, Therneau TM, Rajkumar SV, Offord JR, Larson DR, Plevak MF, et al. A long-term study of prognosis in monoclonal gammopathy of undetermined significance. N Engl J Med. 2002;346:564-9.

4. Leung N, Bridoux F, Hutchison CA, Nasr SH, Cockwell P, Fermand JP, et al. Monoclonal gammopathy of renal significance: when MGUS is no longer undetermined or insignificant. Blood. 2012;120:4292-5.

5. Leung N, Bridoux F, Batuman V, Chaidos A, Cockwell P, D'Agati VD, et al. The evaluation of monoclonal gammopathy of renal significance: a consensus report of the International Kidney and Monoclonal Gammopathy Research Group. Nature Rev Nephrol. 2019;15:45-59.

6. Ciocchini M, Arbelbide J, Musso CG. Monoclonal gammopathy of renal significance (MGRS): the characteristics and significance of a new meta-entity. Int Urol Nephrol. 2017;49:2171-5.

7. Bridoux F, Leung N, Hutchison CA, Touchard G, Sethi S, Fermand JP, et al. Diagnosis of monoclonal gammopathy of renal significance. Kidney Int. 2015;87:698-711.

8. Leung N, Drosou ME, Nasr SH. Dysproteinemias and glomerular disease. CJASN. 2018;13:128-39.

9. Mullens W, Nijst P. Cardiac output and renal dysfunction: definitely more than impaired flow. J Am Coll Cardiol. 2016;67:2209-12.

10. Smith RJ, Appel GB, Blom AM, Cook HT, D'Agati VD, Fakhouri F, et al. C3 glomerulopathy - understanding a rare complement-driven renal disease. Nat Rev Nephrol. 2019;15:129-43.

11. Ravindran A, Fervenza FC, Smith RJ, Sethi S. C3 glomerulopathy associated with monoclonal $\mathrm{Ig}$ is a distinct subtype. Kidney Int. 2018;94:178-86.

12. Pickering MC, D'Agati VD, Nester CM, Smith RJ, Haas M, Appel GB, et al. C3 glomerulopathy: consensus report. Kidney Int. 2013;84:1079-89.

13. Fakhouri F, Fremeaux-Bacchi V, Noel LH, Cook HT, Pickering MC. C3 glomerulopathy: a new classification. Nat Rev Nephrol. 2010;6:494-9.

14. Zand L, Kattah A, Fervenza FC, Smith RJ, Nasr SH, Zhang Y, et al. C3 glomerulonephritis associated with monoclonal gammopathy: a case series. Am J Kidney Dis. 2013;62:506-14.

15. Koopman JJ, Teng YK, Boon CJ, van den Heuvel LP, Rabelink TJ, van Kooten C, et al. Diagnosis and treatment of C3 glomerulopathy in a center of expertise. The Neth J Med. 2019;77:10-8.

16. Zand L, Lorenz EC, Cosio FG, Fervenza FC, Nasr SH, Gandhi MJ, et al. Clinical findings, pathology, and outcomes of C3GN after kidney transplantation. JASN. 2014;25:1110-7.

17. Bomback AS, Appel GB. Pathogenesis of the C3 glomerulopathies and reclassification of MPGN. Nat Rev Nephrol. 2012;8:634-42.

18. Chauvet S, Roumenina LT, Aucouturier P, Marinozzi MC, Dragon-Durey MA, Karras A, et al. Both monoclonal and polyclonal immunoglobulin contingents mediate complement activation in monoclonal gammopathy associated-C3 glomerulopathy. Front Immunol. 2018;9:2260.

19. Sethi S, Rajkumar SV. Monoclonal gammopathy-associated proliferative glomerulonephritis. Mayo Clin Proc. 2013;88:1284-93.

20. Leung N, Barnidge DR, Hutchison CA. Laboratory testing in monoclonal gammopathy of renal significance (MGRS). Clin Chem Lab Med. 2016;54:929-37.

21. Jain A, Haynes R, Kothari J, Khera A, Soares M, Ramasamy K. Pathophysiology and management of monoclonal gammopathy of renal significance. Blood Adv. 2019;3:2409-23.

22. Hogan JJ, Weiss BM. Bridging the divide: an onco-nephrologic approach to the monoclonal gammopathies of renal significance. CJASN. 2016;11:1681-91.

23. Bridoux F, Desport E, Fremeaux-Bacchi V, Chong CF, Gombert JM, Lacombe C, et al. Glomerulonephritis with isolated C3 deposits and monoclonal gammopathy: a fortuitous association? CJASN. 2011;6:2165-74

24. Sethi S, Fervenza FC, Zhang Y, Zand L, Meyer NC, Borsa N, et al. Atypical postinfectious glomerulonephritis is associated with abnormalities in the alternative pathway of complement. Kidney Int. 2013;83:293-9.

25. Larsen CP, Messias NC, Walker PD, Fidler ME, Cornell LD, Hernandez $\mathrm{LH}$, et al. Membranoproliferative glomerulonephritis with masked monotypic immunoglobulin deposits. Kidney Int. 2015;88:867-73.

26. Nasr SH, Fidler ME, Said SM. Paraffin immunofluorescence: a valuable ancillary technique in renal pathology. Kidney Int Rep. 2018;3:1260-6.

27. Lloyd IE, Gallan A, Huston HK, Raphael KL, Miller DV, Revelo MP, et al. C3 glomerulopathy in adults: a distinct patient subset showing frequent association with monoclonal gammopathy and poor renal outcome. Clin Kidney J. 2016;9:794-9.

28. Fermand JP, Bridoux F, Kyle RA, Kastritis E, Weiss BM, Cook MA, et al. How I treat monoclonal gammopathy of renal significance (MGRS). Blood. 2013;122:3583-90.

29. Offidani M, Corvatta L, Marconi M, Malerba L, Mele A, Olivieri A, et al. Common and rare side-effects of low-dose thalidomide in multiple myeloma: focus on the dose-minimizing peripheral neuropathy. Eur $\mathrm{J}$ Haematol. 2004;72:403-9.

30. Steiner N, Gobel G, Suchecki P, Prokop W, Neuwirt H, Gunsilius E. Monoclonal gammopathy of renal significance (MGRS) increases the risk for progression to multiple myeloma: an observational study of 2935 MGUS patients. Oncotarget. 2018;9:2344-56 